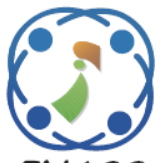

\title{
Estimation of Magnitude and Phase Angle of Power Harmonics Using Demodulation principle of OFDM
}

\author{
Srihari Mandava $^{1} \quad$ Abhishek Gudipalli $^{{ }^{*}} \quad$ Vidhyasagar Gorla $^{1} \quad$ Amutha Prabha Nagarajan $^{1}$ \\ ${ }^{1}$ School of Electrical Engineering, VIT University, Vellore, India \\ * Corresponding author's Email: abhishek.g@vit.ac.in
}

\begin{abstract}
The harmonics injecting into power systems are increasing from day to day with the increasing use of power electronics and nonlinear loads. These harmonics are to be monitored and analyzed to measure their impact on the quality of the power flowing through the lines during normal and abnormal conditions. It is easy to measure the fundamental frequency and it's off nominals of power signal using DFT and its extended methods when the power system frequency is stable. But, with increase in micro-grids and renewable energy sources like wind into the power network, the frequency of the power system in not stable. The DFT leads to errors in frequency estimation during this unstable state. This work uses the demodulation method for estimating the amplitudes and phase angle of fundamental frequency and its off-nominals of a power system having dynamics in frequency. This demodulation principle is part of the orthogonal frequency division multiplexing (OFDM) used for digital multi-carrier modulation.
\end{abstract}

Keywords: Harmonics, Demodulation, FFT, Orthogonal, OFDM.

\section{Introduction}

With the increase in technology, the use of power electronics is increasing in the modern power system networks. Along with this, the use of nonlinear loads is increasing at the load side [1]. The use of this power electronics and the non-linear loads incorporate switching action which produces series of current interruptions. These switching operations gives rise to current which will have not the fundamental component but also the multiples of it. The multiples of the fundamental frequency component are called harmonics [2]. These harmonics affects the power system network steering to increase servicing cost, mal-operation of the protection system or reduces the capability of the power system devices. The principal problem being faced in the microgrid is the change in the frequency and the difficult in controlling it [3]. Along with the change in frequency, the total harmonic distortion in the micro-grid is also increasing.

Many techniques are available in literature for providing the protection and analyzing the power system in which they assume that the frequency of the power system is always stable [4, 5]. Also, various techniques of identifying the off nominal frequencies of signal processing are also used to monitor the signals having the multiples of fundamental frequency in the power system signal. The measured signals are then compensated by some compensating devices and these compensating techniques are completely dependent on the measured frequency component of fundamental and multiples of it. The correct estimation of frequencies is not that much easy and various literature works are present on this which has some margin in estimating the fundamental frequency. The continuous change in the power system configuration, load condition and the operational framework leads to change in the frequency of the harmonic components. So, one of the key point in the modern power system network which is aiming to smart grid in near future is to identify the changes in the fundamental frequency and the multiples of it accurately and make proper adjustments to the protection system and to the monitoring algorithms so that the mal-operations, the servicing costs and 
the derating of the power system components comes down.

The technique used frequently in measuring the frequency of power system is Discrete Fourier Transform (DFT). This is because of its simplicity and lower computational complexity. But, whenever there are changes in the system, the frequency of power system changes. There are other techniques which are proposed in literature some of which are wavelet transforms [6], phase locked loops [7], least square techniques [8], kalman filters [9], neural networks [10], gauss gradient newton methods [11]. The LS and Kalman have proven to be better than fast fourier transform, but when there is a sudden change in amplitude, frequency or phase, these methods are not good enough as they take more time for estimating the parameters of the signal. The proposed technique is based on the demodulation part of OFDM receiver technique for estimating the phase and the amplitude of the fundamental frequency component of the power signal. The demodulation methods used for estimating the fundamental frequency is also presented in [12], [13]. In this paper, the principle of OFDM receiver is used to estimate the harmonics of power signal. In communication, the data transmitted in the form of bits is to be received without any error. Multiple carrier signals are used in OFDM and generate orthogonal frequencies without making use of any oscillatory circuit. The guard band insertion along with the orthogonal frequencies receives the transmitted data efficiently. The demodulation method on the power signal not only estimates the fundamental component but also the harmonics of it. This principle is used as base in power system to estimate the off nominal frequencies and is quite challenging. The sub carriers used in OFDM are considered as harmonics in this work. The harmonics of power signal are orthogonal to each other as like the sub carriers are orthogonal in OFDM technique.

Section 2 gives the problem formulation of the work and section 3 introduces the OFDM principle.

\section{Problem formulation}

Let the power signal from the power system is

$$
x(t)=\sum_{m=1}^{N_{h}} A_{m}(t) \cos \left[m \Omega_{o} t+\emptyset_{m}(t)\right]+\eta(t)
$$

Where $A_{m}(t)$ is the amplitude, $\emptyset_{m}(t)$ is the phase of the m-th harmonic, $N_{h}$ is the maximum harmonic order, $\eta(t)$ is the additive noise, and $\Omega_{o}$ denotes the angular fundamental synchronous frequency. From $[14,15]$, the $\mathrm{m}$-th harmonic frequency is given by

$$
\psi_{m}(t)=\frac{d\left[m \Omega_{o} t+\emptyset_{m}(t)\right]}{d t}=m \Omega_{o}+\frac{d \emptyset_{m}(t)}{d t}
$$

Let $\Omega_{o}$ be constant, so any change in $\psi_{m}(t)$ can be shown by using the term $\frac{d \emptyset_{m}(t)}{d t}$. If $\psi_{m}(t)$ is the $\mathrm{m}$ th harmonic, its frequency is $\mathrm{m}$ times the fundamental frequency i.e. $\psi_{m}(t)=m \psi_{1}(t)$. Substituting this in Eq.(2)

$$
\begin{aligned}
& m \Omega_{o}+\frac{d \emptyset_{m}(t)}{d t}=m\left[\Omega_{o}+\frac{d \emptyset_{1}(t)}{d t}\right. \\
& \therefore \quad \frac{d \emptyset_{m}(t)}{d t}=m \frac{d \emptyset_{1}(t)}{d t}
\end{aligned}
$$

Let the demodulation signal of the k-th harmonic is

$$
\begin{aligned}
d_{c_{k}}(t) & =\cos \left[k \Omega_{o} t+\varphi_{k}(t)\right] \\
\text { and } \quad d_{s_{k}}(t) & =\sin \left[k \Omega_{o} t+\varphi_{k}(t)\right]
\end{aligned}
$$

where $\mathrm{k} \in\left\{1,2, \ldots \ldots, \mathrm{N}_{h}\right\}$ and $\varphi_{k}(t)$ is phase of $d_{c_{k}}(t)$ and $d_{s_{k}}(t)$. The frequencies for both the demodulation signals is then

$$
v_{k}(t)=\frac{d\left[k \Omega_{o} t+\varphi_{k}(t)\right]}{d t}=k \Omega_{o}+\frac{d \varphi_{k}(t)}{d t}
$$

Let $v_{1}(t)$ be the fundamental angular demodulation frequency, then from Eq. (6) $\frac{d \varphi_{k}(t)}{d t}=k \frac{d \varphi_{1}(t)}{d t}$

Multiplying $x(t)$ by Eqs. (4) and (5), $y_{c_{k}}(t)$ and $y_{s_{k}}(t)$ are obtained as

$$
\begin{gathered}
y_{c_{k}}(t)=\sum_{m=1}^{N_{h}} A_{m}(t) \cos \left[m \Omega_{o} t+\emptyset_{m}(t)\right] \cos \left[k \Omega_{o} t\right. \\
\left.\quad+\varphi_{k}(t)\right] \\
\quad+\eta(t) \cos \left[k \Omega_{o} t+\varphi_{k}(t)\right] \\
y_{s_{k}}(t)=\sum_{m=1}^{N_{h}} \begin{array}{l}
A_{m}(t) \cos \left[m \Omega_{o} t\right. \\
\\
\left.+\emptyset_{m}(t)\right] \sin \left[k \Omega_{o} t+\varphi_{k}(t)\right] \\
+\eta(t) \sin \left[k \Omega_{o} t+\varphi_{k}(t)\right]
\end{array}
\end{gathered}
$$

From trigonometric relations, $\quad \cos a \cos b=$ $\frac{1}{2} \cos (a+b)+\frac{1}{2} \cos (a-b), \quad$ and $\quad \sin a \cos b=$ $\frac{1}{2} \sin (a+b)+\frac{1}{2} \sin (a-b)$ in Eqs. (7) and (8), in which $a=k \Omega_{o} t+\varphi_{k}(t)$ and $b=m \Omega_{o} t+\emptyset_{m}(t)$ 
$y_{c_{k}}(t)=\sum_{m=1}^{N_{h}} A_{m}(t) \cos \left[k \Omega_{o} t+\varphi_{k}(t)+\right.$ $\left.m \Omega_{o} t+\emptyset_{m}(t)\right]+\sum_{m=1}^{N_{h}} A_{m}(t) \cos \left[k \Omega_{o} t+\varphi_{k}(t)-\right.$ $\left.m \Omega_{o} t+\emptyset_{m}(t)\right]+\eta(t) \cos \left[k \Omega_{o} t+\varphi_{k}(t)\right]$

$y_{s_{k}}(t)=\sum_{m=1}^{N_{h}} A_{m}(t) \sin \left[k \Omega_{o} t+\varphi_{k}(t)+\right.$ $\left.m \Omega_{o} t+\emptyset_{m}(t)\right]+\sum_{m=1}^{N_{h}} A_{m}(t) \sin \left[k \Omega_{o} t+\right.$ $\left.\varphi_{k}(t)-m \Omega_{o} t-\emptyset_{m}(t)\right]+\eta(t) \sin \left[k \Omega_{o} t+\right.$ $\left.\varphi_{k}(t)\right]$

Thus,

$$
\begin{aligned}
& y_{c_{k}}(t)=\sum_{m=1}^{N_{h}} \frac{1}{2} A_{m}(t) \cos \left[m \Omega_{o} t+k \Omega_{o} t+\right. \\
& \left.\emptyset_{m}(t)+\varphi_{k}(t)\right]+\sum_{m=1}^{N_{h}} \frac{1}{2} A_{m}(t) \cos \left[m \Omega_{o} t-\right. \\
& \left.k \Omega_{o} t+\emptyset_{m}(t)-\varphi_{k}(t)\right]+\eta(t) \cos \left[k \Omega_{o} t+\right. \\
& \left.\varphi_{k}(t)\right]
\end{aligned}
$$

$$
\begin{aligned}
& y_{s_{k}}(t)=\sum_{m=1}^{N_{h}} \frac{1}{2} A_{m}(t) \sin \left[m \Omega_{o} t+k \Omega_{o} t+\right. \\
& \left.\emptyset_{m}(t)+\varphi_{k}(t)\right]-\sum_{m=1}^{N_{h}} \frac{1}{2} A_{m}(t) \sin \left[m \Omega_{o} t-\right. \\
& \left.k \Omega_{o} t+\emptyset_{m}(t)-\varphi_{k}(t)\right]+\eta(t) \sin \left[k \Omega_{o} t+\right. \\
& \left.\varphi_{k}(t)\right]
\end{aligned}
$$

For any value of $k$, the frequencies of first and second terms of both signals of Eqs. (14) and (15) are

$$
\begin{aligned}
& \varphi_{k}(t)=m \Omega_{o}+k \Omega_{o}+\frac{d \emptyset_{m}(t)}{d t}+\frac{d \varphi_{k}(t)}{d t} \\
& \psi_{k}(t)=m \Omega_{o}+k \Omega_{o}+\frac{d \emptyset_{m}(t)}{d t}-\frac{d \varphi_{k}(t)}{d t}
\end{aligned}
$$

for $\mathrm{m}=1,2, \ldots \ldots \ldots, \mathrm{N}_{h}$.

Substituting Eqs. (3) and (7) in Eqs. (13), (14)

$$
\begin{aligned}
& \emptyset_{k}(t)=(m+k) \Omega_{o}+m \frac{d \emptyset_{m}(t)}{d t}+k \frac{d \varphi_{k}(t)}{d t} \\
& \psi_{k}(t)=(m-k) \Omega_{o}+m \frac{d \emptyset_{m}(t)}{d t}-k \frac{d \varphi_{k}(t)}{d t}
\end{aligned}
$$

for $\mathrm{m}=1,2, \ldots \ldots \ldots, \mathrm{N}_{h}$. If $\frac{d \varphi_{k}(t)}{d t}$ is an estimate of $\frac{d \emptyset_{k}(t)}{d t},(15)$ and (16) will be

$$
\begin{aligned}
& \emptyset_{k}(t) \cong(m+k)\left[\Omega_{o}+\frac{d \emptyset_{1}(t)}{d t}\right]=(m+k) \psi_{1}(t) \\
& \psi_{k}(t) \cong(m-k)\left[\Omega_{o}+\frac{d \emptyset_{1}(t)}{d t}\right]=(m-k) \psi_{1}(t)
\end{aligned}
$$

For $m=1,2, \ldots \ldots \ldots, \mathrm{N}_{h} . \quad$ As, $(\mathrm{m}-\mathrm{k}) \quad$ can assume negative values in Eq. $(18), \psi_{k}(t)$ can also be negative. To work with only positive values, the second term of Eqs. (12) and (13) is taken as $\left|\psi_{k}(t)\right|$ for the frequencies. From $\emptyset_{k}(t)$ and $\left|\psi_{k}(t)\right|$, it can be said that $y_{c_{k}}(t)$ and $y_{s_{k}}(t)$ will consist of a constant current i.e. the DC value (at $\mathrm{m}=\mathrm{k})$ and frequency components at $\psi_{1}(t), 2 \psi_{1}(t), 3 \psi_{1}(t) \ldots \ldots, \max \left(\mathrm{k}-1, \mathrm{~N}_{h}-\mathrm{k}\right) \psi_{1}(t)$, $(\mathrm{k}+1) \psi_{1}(t),(\mathrm{k}+2) \psi_{1}(t), \ldots \ldots,\left(\mathrm{N}_{h}+1\right) \psi_{1}(t)$. Using low pass filter on $y_{c_{k}}(t)$ and $y_{s_{k}}(t)$ gives

$$
\begin{gathered}
\psi_{c c_{k}}(t)=\frac{1}{2} A_{k}(t) \cos \left[\emptyset_{k}(t)-\varphi_{k}(t)\right]+\eta_{c}(t) \\
\psi_{s s_{k}}(t)=-\frac{1}{2} A_{k}(t) \sin \left[\emptyset_{k}(t)-\varphi_{k}(t)\right]+\eta_{s}(t)
\end{gathered}
$$

Where $\eta_{c}(t)$ and $\eta_{s}(t)$ are respectively the low pass component values of $\eta(t) \cos \left[k \Omega_{o} t+\emptyset_{d_{k}}(t)\right]$ and $\eta(t) \sin \left[k \Omega_{o} t+\emptyset_{d_{k}}(t)\right]$. If the low pass filter possess good attenuation in the stop band, the $\eta_{c}(t)$ and $\eta_{s}(t)$ are negligible and hence the value of amplitude and phase of the k-th order harmonic component are estimated using

$$
\begin{aligned}
& \hat{A}_{k}(t)=2 \sqrt{y_{c c_{k}}^{2}(t)+y_{s s_{k}}^{2}(t)} \\
& \widehat{\emptyset}_{k}(t)=-\arctan \left[\frac{y_{s s_{k}}(t)}{y_{c c_{k}}(t)}\right]+\varphi_{k}(t)
\end{aligned}
$$

In power systems, the phase angle difference between two signals is given by [16] (22), in which $\widehat{\emptyset_{k_{a}}}(t)$ and

$$
\Delta \widehat{\emptyset}_{k}(t)=\widehat{\emptyset_{k_{a}}}(t)-\widehat{\emptyset_{k_{b}}}(t)
$$

$\widehat{\emptyset_{k_{b}}}(t)$ are the phase values of $x_{a}(t)$ and $x_{b}(t)$. But, in an present modern interconnected power system, the demodulating signal is same for all signals and so, the $\widehat{\emptyset}_{k}(t)$ will be independent of $\varphi_{k}(t)$. Hence, $\varphi_{k}(t)$ is subtracted from Eq. (21) as this won't affect the phase difference. So, $\widehat{\emptyset}_{k}(t)$ is given by

$$
\widehat{\emptyset}_{k}(t)=-\arctan \left[\frac{y_{s s_{k}}(t)}{y_{c c_{k}}(t)}\right]
$$

The block diagram of the technique presented so far is shown in Fig. 1. The LPF are the low pass filters and the cos and sin gives the demodulation signals expressed by Eqs. (4) and (5). The Amplitude and Phase are the values of the expressions (21) and (24). 


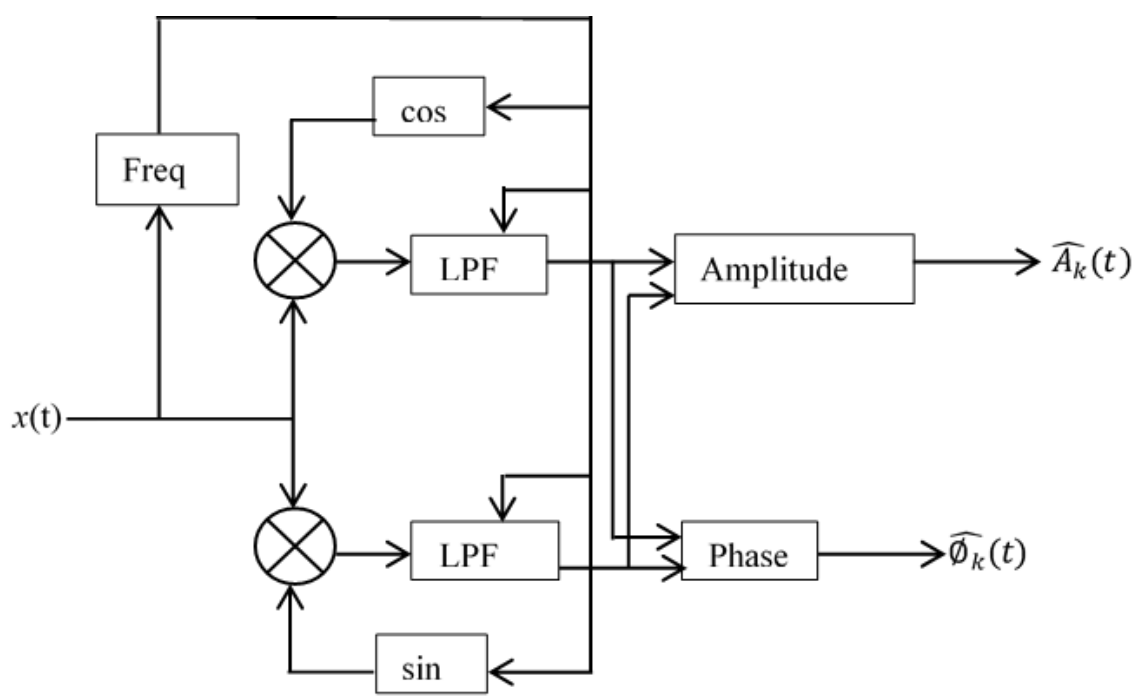

Figure. 1 Modulation principle

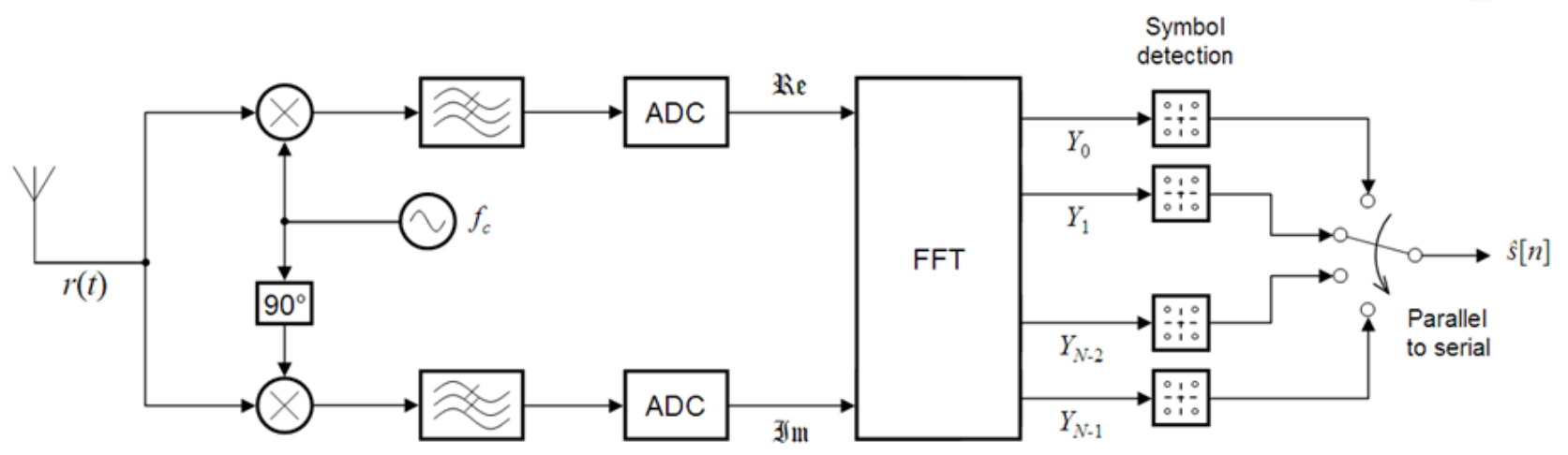

Figure. 2 OFDM receiver

In this, the demodulation signals are controlled and the frequency of the low pass filters by the Freq block. This demodulation method is different from the prior methods in two ways.

i) The Sin and Cos block are fixed in the old methods where as these blocks vary in this method according to the frequency value.

ii) The low-pass filter coefficients vary in this method while they remain same in the old methods.

This technique is a part of the OFDM receiver principle. The OFDM receiver is as shown in Fig. 2. The real and the imaginary values of Fig. 2 are the amplitude and phase values of Fig. 1. The amplitude is the magnitude of harmonic signal and the phase value is the angle of the harmonic signal. Then, the FFT is implemented on the amplitude and the plot of this gives the frequency of the harmonic.

\section{Basic principle of OFDM principle and application to power system}

OFDM principle is used mostly in $3 \mathrm{G}$ system to take off the signal attenuation problem. The main function of OFDM technique is to reduce the multi path transmission using multi carrier system and proper guard band. This principle of OFDM is modified for analyzing the power system off nominal frequencies. In communication, the data transmitted in the form of bits is to be received without any error. The OFDM uses multiple carriers and generate orthogonal frequencies without making use of any oscillatory circuit. The guard band insertion along with the orthogonal frequencies receives the transmitted data efficiently. This principle is used as base in power system to estimate the off nominal frequencies and is quite challenging. The sub carriers used in OFDM are considered as harmonics in this work. The harmonics are orthogonal to each other as like the sub carriers are orthogonal in OFDM technique.

In OFDM, inverse fast fourier transform (IFFT) is used in transmitter and fast fourier transform (FFT) is used in receiver part. This work makes use of the receiver part of actual OFDM technique and the transmitter part of OFDM is considered as a power signal containing harmonics. The harmonics 


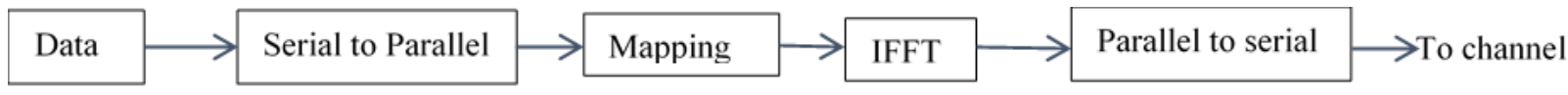

Figure. 3 OFDM transmitter

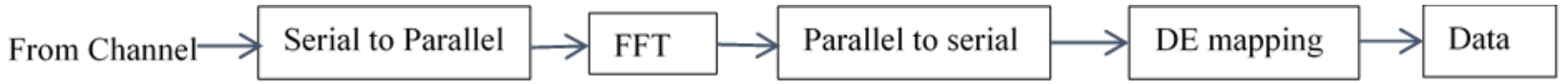

Figure. 4 OFDM receiver

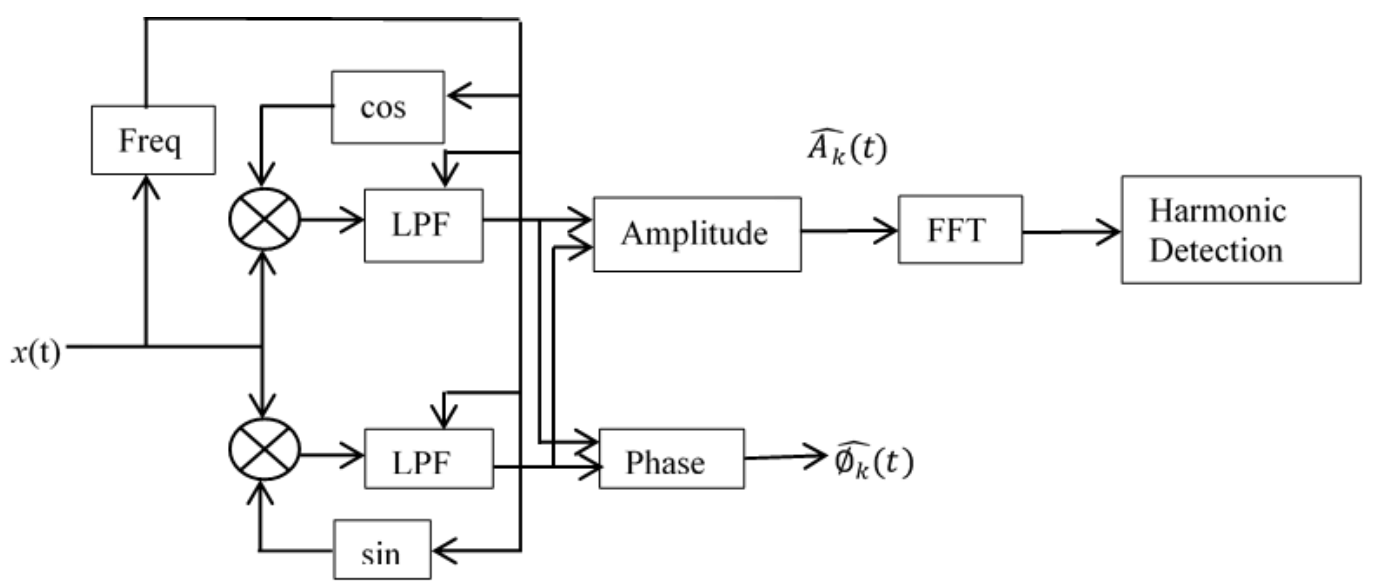

Figure. 5 Proposed OFDM receiver principle for harmonic detection

of power signal are nothing but various sub carriers in OFDM. The sub carriers in the OFDM are estimated at the receiving end which is equal to estimation of harmonics using FFT in power line. OFDM has two modulation stages, the base band modulation having many sub carriers and carrier modulation having only one frequency. The frequency in the carrier modulation is higher than the sub carrier frequencies of the base band modulation. Since, the fundamental frequency of Indian power system is $50 \mathrm{~Hz}$, the harmonics are viewed as base band modulation using many orthogonal frequency signals. The actual OFDM transmitter and receiver are as shown in Fig. 3 and Fig. 4.

\section{Technique implementation}

The expression (1) in discrete form is

$$
x[n]=\sum_{m=1}^{N_{h}} A_{m}[n] \cos \left[m \omega_{o} t+\emptyset_{m}[n]\right]+\eta[n]
$$

Where $\omega_{o}=\frac{\Omega_{o}}{f_{s}}$ is the synchronous angular frequency in discrete mode and $f_{S}$ is the sampling rate. The demodulation signals from expression (4) and (5) are given by Eqs. (26) and (27).

$$
d_{c_{k}}[n]=\cos \left[k \Omega_{o} n+\varphi_{k}[n]\right]
$$

$$
\text { and } \quad d_{s_{k}}(t)=\sin \left[k \Omega_{o} n+\varphi_{k}[n]\right]
$$

The discrete version of continuous derivative is

$$
\frac{d \varphi_{k}(t)}{d t}<=>f_{S}\left(\varphi_{k}[n]-\varphi_{k}[n-1]\right)
$$

The discrete version of Eq. (6) for the demodulation frequency is given by

$$
v_{k}[n]=k \Omega_{o}+f_{s}\left(\varphi_{k}[n]-\varphi_{k}[n-1]\right)
$$

Let the demodulation frequency of Eq. (29) be $v_{k}[n]=k v_{1}[n]$ which is the frequency of $k \psi_{1}[n]$. So, from Eq. (29)

$$
\varphi_{k}[n]=\varphi_{k}[n-1]+\frac{k}{f_{s}}\left(\psi_{1}[n]-\Omega_{o}\right)
$$

From (30), it is clear that $\psi_{1}[n]$, the fundamental frequency of signal $x(n)$ is identified, then the value of phase $\varphi_{k}[n]$ of the demodulation signals is easily modified for each sample period. Then, the frequency of both the demodulation signals is changed to match the frequency of the respective harmonic. Several approaches are present for finding $\psi_{1}[n]$ and in this work, phase locked loop (PLL) is used to identify it.

Generally, the power system frequency will not remain constant and changes slowly in time. This change in frequency will change the harmonic components in the same proportion. By using this technique, these changes are clearly observed. The 
final diagram of the logic used to identify the harmonics is shown in Fig. 4. The technique of Fig. 5 resembles the OFDM receiver technique shown in Fig. 2.

\section{Results}

The sampling frequency is taken as $6400 \mathrm{~Hz}$. The test signal considered is

$$
\begin{aligned}
& x[n]=A_{1}[n]\left.\cos \left(\omega_{0} n+\emptyset_{1}[n]\right)\right] \\
&+ A_{3}[n] \cos \left(3 \omega_{0} n+\emptyset_{3}[n]\right) \\
&+ A_{5}[n] \cos \left(5 \omega_{0} n+\emptyset_{5}[n]\right) \\
&+A_{7}[n] \cos \left(7 \omega_{0} n+\emptyset_{1}[n]\right) \\
&+A_{9}[n] \cos \left(9 \omega_{0} n+\emptyset_{9}[n]\right) \\
&+A_{11}[n] \cos \left(11 \omega_{11} n+\emptyset_{11}[n]\right) \\
&+A_{13}[n] \cos \left(13 \omega_{0} n+\emptyset_{13}[n]\right) \\
&+A_{15}[n] \cos \left(15 \omega_{0} n+\emptyset_{15}[n]\right) \\
&+A_{17}[n] \cos \left(17 \omega_{0} n+\emptyset_{17}[n]\right) \\
&+A_{19}[n] \cos \left(19 \omega_{0} n+\emptyset_{19}[n]\right) \\
&+A_{21}[n] \cos \left(21 \omega_{0} n+\emptyset_{21}[n]\right) \\
&+A_{23}[n] \cos \left(23 \omega_{0} n+\emptyset_{23}[n]\right) \\
&+A_{25}[n] \cos \left(25 \omega_{0} n+\emptyset_{25}[n]\right) \\
&+A_{27}[n] \cos \left(27 \omega_{0} n+\emptyset_{27}[n]\right) \\
&\left.+A_{29}[n] \cos \left(29 \omega_{0} n+\emptyset_{29}[n]\right)\right]
\end{aligned}
$$

Where $\omega_{0}=\frac{2 \pi 50}{f_{s}}$. The amplitudes and phases considered for each harmonic are as shown in Table .1. The phase angles and the amplitudes of up to 29th order the harmonics obtained after implementing the OFDM principle is as shown in Fig. 6 and Fig. 7. The magnitude and phase angles of each harmonic signal is approximately same with the given table values.

Table 1. Amplitudes and Phase angles for each harmonic signal

\begin{tabular}{|c|c|c|c|}
\hline \multicolumn{2}{|c|}{ Amplitude } & \multicolumn{2}{c|}{ Phase angle } \\
\hline $\mathrm{A} 1[\mathrm{n}]$ & 1 & $\emptyset_{1}[n]$ & -3.0 \\
\hline $\mathrm{A} 3[\mathrm{n}]$ & $1 / 3$ & $\emptyset_{3}[n]$ & -2.5 \\
\hline $\mathrm{A} 5[\mathrm{n}]$ & $1 / 5$ & $\emptyset_{5}[n]$ & -2.0 \\
\hline $\mathrm{A} 7[\mathrm{n}]$ & $1 / 7$ & $\emptyset_{7}[n]$ & -1.5 \\
\hline $\mathrm{A} 9[\mathrm{n}]$ & $1 / 9$ & $\emptyset_{9}[n]$ & -1.0 \\
\hline $\mathrm{A} 11[\mathrm{n}]$ & $1 / 11$ & $\emptyset_{11}[n]$ & -0.5 \\
\hline $\mathrm{A} 13[\mathrm{n}]$ & $1 / 13$ & $\emptyset_{12}[n]$ & 0.0 \\
\hline $\mathrm{A} 15[\mathrm{n}]$ & $1 / 15$ & $\emptyset_{13}[n]$ & 0.5 \\
\hline $\mathrm{A} 17[\mathrm{n}]$ & $1 / 17$ & $\emptyset_{14}[n]$ & 1.0 \\
\hline $\mathrm{A} 19[\mathrm{n}]$ & $1 / 19$ & $\emptyset_{15}[n]$ & 1.5 \\
\hline $\mathrm{A} 21[\mathrm{n}]$ & $1 / 21$ & $\emptyset_{15}[n]$ & 2.0 \\
\hline $\mathrm{A} 23[\mathrm{n}]$ & $1 / 23$ & $\emptyset_{19}[n]$ & 2.5 \\
\hline $\mathrm{A} 25[\mathrm{n}]$ & $1 / 25$ & $\emptyset_{21}[n]$ & 3.0 \\
\hline $\mathrm{A} 27[\mathrm{n}]$ & $1 / 27$ & $\emptyset_{22}[n]$ & 3.5 \\
\hline $\mathrm{A} 29[\mathrm{n}]$ & $1 / 29$ & $\emptyset_{23}[n]$ & 4.0 \\
\hline
\end{tabular}

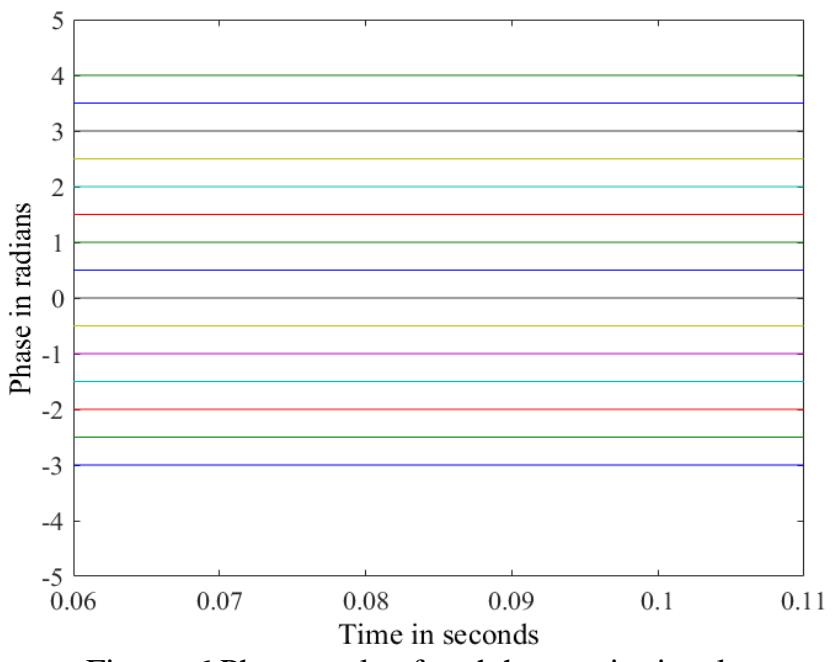

Figure. 6 Phase angle of each harmonic signal

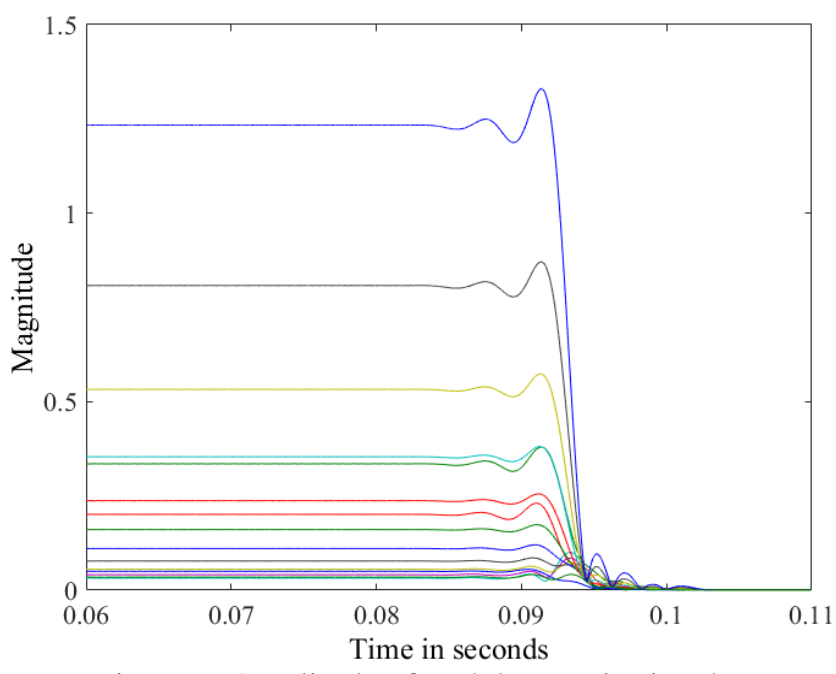

Figure. 7 Amplitude of each harmonic signal

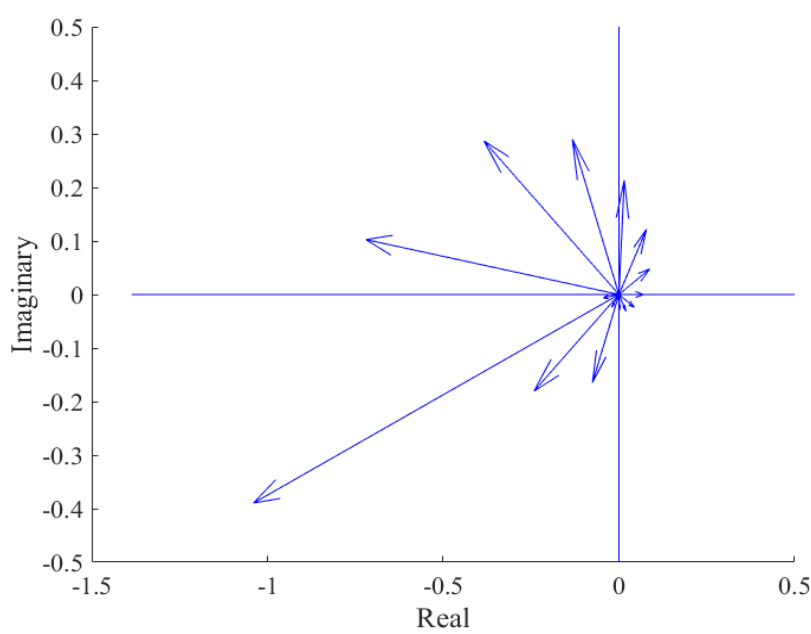

The phasor plot of all the harmonics is shown in the Fig. 8.The $\mathrm{fft}$ is applied to the amplitude of all the harmonics and the fft plot for the harmonics 7th, 13th and 17th is shown in the Fig. 9. The frequency observed from the $\mathrm{fft}$ plot is matching with the frequency of the harmonic signal. 


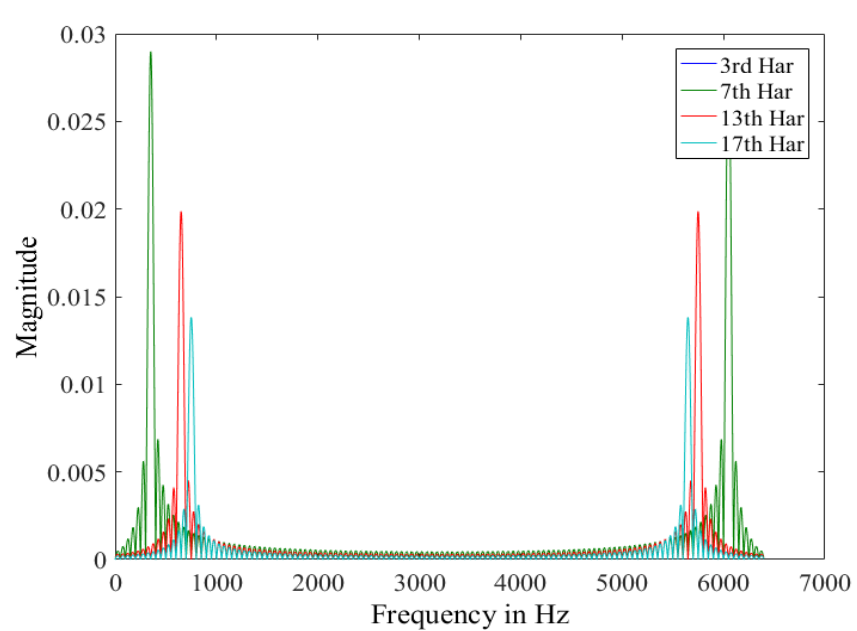

Figure. 9 FFT plot of amplitude for $7^{\text {th }}, 13^{\text {th }}, 17^{\text {th }}$ harmonic signal

\section{Conclusion}

In this work, the OFDM receiver principle is successfully implemented for detecting the harmonics of the power signal. A novel demodulation technique is introduced for detecting harmonics which is a part of OFDM receiver. The outputs, amplitude and phase angles of each harmonic signal are almost matching with the harmonic input values. The phasor plot of each harmonic is also plotted and then the FFT plot of the Amplitude for all the harmonics has given the frequency value for each harmonic signal. The frequency identified through FFT plot exactly matched with the input harmonic frequency. The noise parameter is not considered in this work. Hence noise can be added to the power signal and analysis can be done on the effect of noise on the measurement of harmonic signal and its parameters.

\section{References}

[1] M.I.A. Bakar, "Assessments for the Impact of Harmonic Current Distortion of Non Linear Load in Power System Harmonics", In: Proc. of the IEEE/PES Transmission and Distribution Conference and Exposition: Latin America, pp.1-6, 2008.

[2] N. R. Watson and J. Arrigala, "Harmonics in large systems", Electrical Power Systems Res., Vol. 66, pp.15-29, 2003.

[3] R. Majumder, A. Ghosh, and G. Ledwich, "Load Frequency Control in a Microgrid: Challenges and Improvements", Smart Power Grids, pp.49-82, 2011.

[4] L.L. Lai, W.L. Chan, C.T. Tse, and A.T.P. So, "Real-time frequency and harmonic evaluation using artificial neural networks", IEEE
Transactions on Power Delivery, Vol.14, No.1, pp.52-59, 1999.

[5] L.M. Tolbert, H.D. Hollis, and P.S. Hale, Jr, "Survey of Harmonics Measurements in Electrical Distribution Systems", In: Proc. of the IEEE IAS Annual Meeting, San Diego, CA, pp.2333-2339, 1996.

[6] W. Jianze, J. Yanchao, W. Fei, and R. Qiwen, "New method for transient harmonics measurement based on wavelet transform", In: Proc. of 24th Annual Conference on IEEE Inustrial Electroics Society, Vol. 2, pp. 636639, 1998.

[7] M.K. Ghartemani and M.R. Iravani, "Measurement of harmonics/interharmonics of time-varying frequencies", IEEE Transactions on Power Delivery, Vol. 20, No. 1, pp.23-31, 2005.

[8] C. Rakpenthai, S. Uatrongjit, N.R. Watson, and S. Premrudeepreechacharn, "On harmonic state estimation of power system with uncertain network parameters", IEEE Transactions on Power Systems, Vol. 28, No. 4, pp. 4829-4838, 2013.

[9] F.F. Costa, A.J.M. Cardoso, and D.A. Fernandes, "Harmonic analysis based on Kalman filtering and Prony's method", In: Proc. of International Conference on Power Engineering and Power Electronics Drives, pp.696-701, 2007.

[10] P. K. Dash, D. P. Swain, A. Routray, and A. C. Liew, "Harmonic estimation in a power system using adaptive perceptrons", IEEE Proceedings on General Transmission and Distribution, Vol. 143, No. 6, pp. 565-574, 1996.

[11] H. Sun, G. H. Allen, and G. D. Cain, "A new filter-bank configuration for harmonic measurement", IEEE Transactions on Instrumentation and Measurements, Vol.45, No.3, pp. 739-744, 1996.

[12] I. Kamwa, M. Leclerc, and D. McNabb, "Performance of demodulation based frequency measurement algorithms used in typical PMUs", IEEE Transactions on Power Delivery, Vol.19, No.2, pp.505-514, 2004.

[13] M. Akke, "Frequency estimation by demodulation of two complex signals", IEEE Transactions on Power Delivery, Vol. 12, No. 1, pp. 157-163, 1997.

[14] M. M. Begović, P. M. Djurić, S. Dunlap, and A. G. Phadke, "Frequency tracking in power networks in the presence of harmonics," IEEE Transactions on Power Delivery, Vol. 8, No. 2, pp. 480-486, 1993. 
[15] B. Boashash, "Estimating and interpreting the instantaneous frequency of a signal. II. Algorithms and applications", In: Proc. of the IEEE, Vol. 80, No. 4, pp.540-568, 1992.

[16] P. M. Djurić, M. M. Begović, and M. Doroslova ki, "Instantaneous phase tracking in power networks by demodulation", IEEE Transactions on Instrumentation and Measurements, Vol. 41, No. 6, pp. 963-967, 1992. 